\title{
Hepatitis B Virus (HBV) Sequence Variation in Cytotoxic T Lymphocyte Epitopes is not Common in Patients with Chronic HBV Infection
}

Barbara Rehermann, Claudio Pasquinelli, Sarah M. Mosier, and Francis V. Chisari

Department of Molecular and Experimental Medicine, The Scripps Research Institute, La Jolla, California 92037

\begin{abstract}
It has been suggested that immune selection pressure exerted by the cytotoxic $T$ lymphocyte (CTL) response could be responsible for viral persistence during chronic hepatitis $B$ virus infection. To address this question, in the current study we compared the DNA and amino acid sequences of, and the CTL responses to, multiple HLA-A2-restricted CTL epitopes in the hepatitis B virus in several HLA-A2positive patients with acute and chronic hepatitis. Our results indicate that the CTL response to these epitopes is barely detectable in the majority of patients with chronic hepatitis. Further, we show that the weak CTL response is not secondary to infection by mutant viruses lacking these epitopes, and we show that the CTL response did not select for escape mutants in any of these patients. We conclude that an ineffective hepatitis B virus specific CTL response is the primary determinant of viral persistence in chronic hepatitis and that immune selection of viral variants is not a common event in the majority of patients. (J. Clin. Invest. 1995. 96:1527-1534.) Key words: hepatitis B virus $•$ immune response $\cdot$ mutation $\cdot$ cytotoxic $\mathbf{T}$ cell $\cdot$ viral escape
\end{abstract}

\section{Introduction}

The hepatitis B virus (HBV) ${ }^{1}$ is a noncytopathic DNA virus with a small, circular DNA genome that causes acute and chronic liver disease and hepatocellular carcinoma. Patients with acute, self-limited HBV infection usually mount a vigorous and polyclonal cytotoxic $T$ cell response, which is also multispecific, i.e., targeted against multiple epitopes in the viral nucleocapsid (1-4), envelope (5) and polymerase proteins (6). In contrast, a vigorous, polyclonal cytotoxic T lymphocyte (CTL) response is usually not readily detectable in the peripheral blood of patients with chronic $\mathrm{HBV}$ infection although HBV-specific $T$ cells are present in the chronically inflamed liver and are thought to contribute to the pathogenesis of chronic hepatitis $(7,8)$. For these reasons it is likely that a strong, polyclonal CTL response to HBV plays an essential role in viral clearance

Address correspondence to Francis V. Chisari, Department of Molecular and Experimental Medicine, SBR-10, The Scripps Research Institute, 10666 North Torrey Pines Road, La Jolla, CA 92037. Phone: 619-5548228; FAX: 619-554-6134.

Received for publication 7 February 1995 and accepted in revised form.18 May 1995.

1. Abbreviations used in this paper: CTL, cytotoxic T lymphocyte; HBV, hepatitis B virus; LCMV, lymphocytic choriomeningitis virus.

J. Clin. Invest.

(c) The American Society for Clinical Investigation, Inc.

0021-9738/95/09/1527/08 \$2.00

Volume 96, September 1995, 1527-1534 and clinical recovery during acute hepatitis, and that viral persistence reflects a diminished CTL response that is not strong enough to clear the virus but is sufficient to maintain the spectrum of low grade inflammatory liver diseases designated as chronic hepatitis.

The host-virus interactions that facilitate viral persistence and cause chronic hepatitis, however, are not well defined. In infected newborns the diminished antiviral immune response is probably due to neonatal tolerance mechanisms, while immunogenetic, environmental, or virological factors could be responsible for the diminished immune response in chronically infected adults. Irrespective of the cause, however, a diminished CTL response that is permissive of viral persistence could, theoretically, select for the emergence of viral escape mutants that are even less visible to the already ineffective immune system and, thereby, contribute further to persistent infection. The likelihood of this process would be significantly enhanced if the CTL response was narrowly focussed and relatively strong (i.e., functionally monospecific) as has been reported to occur during lymphocytic choriomeningitis virus infection in transgenic mice that express a single $\mathrm{T}$ cell receptor specific for a single lymphocytic choriomeningitis virus (LCMV) epitope (9). Importantly, this scenario was recently reported in two patients with chronic $\mathrm{HBV}$ infection who produced a relatively strong, but narrowly focussed CTL response to a single epitope in the hepatitis B virus $(10,11)$.

Several other groups (12-14) have recently described HBV sequence heterogeneity in chronically infected patients, and they have suggested that the sequence heterogeneity could reflect immune selection. However, a systematic comparative analysis of the $T$ cell response to epitopes potentially encoded by these sequences was not performed in these patients, so the significance of those findings is uncertain.

The current study was performed to compare these virological and immunological events simultaneously in a group of patients with chronic HBV infection to clarify the importance of CTL selection pressure and viral escape mutations in this disease.

\section{Methods}

Patient population. 12 HLA-A2-positive patients with chronic hepatitis B and 6 HLA-A2-positive patients with acute hepatitis B were studied. The diagnosis of acute hepatitis $B$ was based on clinical and biochemical evidence of acute liver injury according to standard diagnostic criteria, i.e., jaundice and elevated values of alanine aminotransferase activity at least 20-fold greater than the upper limit of normal, together with serological evidence of acute HBV infection, i.e., hepatitis B surface antigen ( $\mathrm{HBsAg})$, hepatitis $\mathrm{B}$ e antigen $(\mathrm{HBeAg})$ and $\mathrm{IgM}$ anti-HBc antibody (IgM HBc-Ab), and the absence of serologic evidence of hepatitis delta or hepatitis $\mathrm{C}$ virus infection (Abbott Laboratories, North Chicago, IL). All patients recovered completely with normalization of serum transaminases and clearance of $\mathrm{HBsAg}$ and $\mathrm{HBeAg}$ within $4 \mathrm{mo}$ of initial diagnosis. Blood samples for serum and PBMC analysis were taken at the time of clinical presentation, when diagnosis of acute HBV 
Table I. Clinical, Serological, and Virological Features of the Patients Studied

\begin{tabular}{|c|c|c|c|c|c|c|c|c|c|}
\hline Patient* & Age & $\operatorname{Sex}^{\ddagger}$ & HLA & HBsAg & $\mathrm{HBeAg}$ & antiHBc & antiHBs & antiHBe & $\mathrm{ALT}^{3}$ \\
\hline & $y r$ & & & & & & & & $U /$ \\
\hline C-1 & 35 & $\mathbf{M}$ & A2A24 B44B67 & pos. & pos. & pos. & neg. & neg. & 316 \\
\hline C-2 & 38 & $\mathbf{M}$ & A2A30 B44B13 Cw6 & pos. & neg. & pos. & neg. & neg. & 38 \\
\hline $\mathrm{C}-3$ & 36 & $\mathbf{M}$ & A2A69 B41B52 & pos. & pos. & pos. & neg. & neg. & 139 \\
\hline C-4 & 36 & $\mathbf{M}$ & A2 B13B7801 Cw7 & pos. & pos. & pos. & neg. & neg. & 115 \\
\hline C-5 & 49 & $\mathbf{M}$ & A2 A23 B44 & pos. & pos. & pos. & neg. & neg. & 49 \\
\hline C-6 & 66 & $\mathbf{M}$ & A2A1 B8B44 & pos. & pos. & pos. & neg. & neg. & 123 \\
\hline C-7 & 21 & $\mathbf{F}$ & A2A26 B46B72 Cw3Cw1 & pos. & neg. & pos. & pos. & neg. & 105 \\
\hline C-8 & 57 & $\mathbf{M}$ & A2A68 B59B44 Cw5Cw7 & pos. & pos. & pos. & neg. & neg. & 78 \\
\hline C-9 & n.k." & $\mathbf{M}$ & A2 B62 Cw8 & pos. & pos. & pos. & pos. & neg. & 79 \\
\hline C-10 & 53 & $\mathbf{M}$ & A2A34 B8B27 Cw7 & pos. & pos. & pos. & neg. & neg. & 120 \\
\hline C-11 & 50 & $\mathbf{M}$ & A2 B27B46 Cw1 & pos. & pos. & pos. & neg. & neg. & 54 \\
\hline C-12 & n.k. & $\mathrm{F}$ & A2 B7B39 & pos. & neg. & pos. & neg. & neg. & 24 \\
\hline A-1 & 26 & $\mathbf{M}$ & A2A1 B8Bw58 Cw7 & pos. & pos. & pos. & n.d. & n.d. & 2082 \\
\hline A-2 & 22 & $\mathbf{M}$ & A2Aw69 Bw53 Cw4 & pos. & pos. & pos. & n.d. & n.d. & 3918 \\
\hline A-3 & 38 & $\mathbf{M}$ & A2A24 B51B53 Cw1 & pos. & pos. & pos. & neg. & neg. & 844 \\
\hline A-4 & 19 & n.k. & A2A24 B7B27 Cw2Cw7 & pos. & n.d. & pos. & n.d. & n.d. & 2044 \\
\hline A-5 & 34 & $\mathbf{M}$ & A2A68 B44B54 Cw5Cw7 & pos. & neg. & pos. & neg. & neg. & 3822 \\
\hline A-6 & 22 & $\mathbf{F}$ & A2A74B62B57Cw3Cw6 & neg. & neg. & pos. & neg. & pos. & 371 \\
\hline
\end{tabular}

* Patients with chronic hepatitis $\mathrm{B}$ are identified by a $\mathrm{C}$ preceding the patient number, patients with acute hepatitis $\mathrm{B}$ with an A; ${ }^{\dagger} \mathrm{M}$, male; $\mathrm{F}$, female; ${ }^{8}$ ALT, alanine aminotransferase, normal range 0-45 U/liter; "I n.k., not known; ' n.d., not done; pos., positive; neg., negative.

infection was first established. All patients with chronic hepatitis B were repeatedly serologically positive for $\mathrm{HBsAg}$ for at least $6 \mathrm{mo}$ and in selected cases (C-1, C-3, C-6) for more than $3 \mathrm{yr}$. Eight of them displayed elevated transaminases of twice the upper limit of the normal range or higher, while four patients (C-2, C-5, C-11, C-12) had mildly elevated or normal transaminases when blood samples for sequencing and CTL analysis were taken. The clinical, virological and serological characteristics of these patients are summarized in Table $\mathbf{I}$.

All subjects studied were repeatedly negative for antibodies to HCV and HIV. HLA typing of PBMC was performed by complement microcytotoxicity using HLA typing trays purchased from One Lambda (Canoga Park, CA) (Table I). The study protocol was approved by the Human Subjects Committee at the Scripps Clinic and Research Foundation.

CTL analysis. PBMC were separated on Ficoll-Histopaque density gradients (Sigma Chemical Co., St. Louis, MO), washed three times in HBSS (GIBCO Laboratories, Grand Island, NY), resuspended in RPMI 1640 (GIBCO Laboratories) supplemented with L-glutamine (2 $\mathrm{mM})$, gentamycin $(10 \mathrm{mM})$, and $10 \%$ heat-inactivated human $\mathrm{AB}$ serum and plated in a 24-well plate at $4 \times 10^{6}$ cells/well. rHBcAg (Biogen, Cambridge, MA) was added to the cell cultures at $1 \mathrm{mM}$ and the synthetic peptides at $10 \mu \mathrm{M}$. The peptides used have been described as CTL-epitopes in previous studies $(3,5,6$, Chisari, F. V., unpublished observations). On days 3 and $10,1 \mathrm{ml}$ of RPMI 1640 with $10 \%$ human $\mathrm{AB}$ serum and rIL-2 (Hoffmann-La Roche, Inc., Nutley, NJ) at $10 \mathrm{U} /$ $\mathrm{ml}$ final concentration was added to each well. On day 7 , the cultures were restimulated with peptide, rIL-2, and irradiated (3,000 rad) autologous feeder cells. In a subset of chronically infected patients the CTL response to an additional panel of $32 \mathrm{HBV}$-derived peptides with good HLA-A2-binding affinity was tested using a modification of the foregoing stimulation strategy as previously described (10). In all cases, cultures were tested for cytotoxic activity on day 14 against JY-EBV incubated overnight with synthetic peptides at $10 \mu \mathrm{M}$, labeled with $100 \mu \mathrm{Ci}$ of ${ }^{51} \mathrm{Cr}$ (Amersham Corp., Arlington Heights, IL) for $1 \mathrm{~h}$ and washed four times with HBSS. Cytolytic activity was determined in a standard 4- ${ }^{51} \mathrm{Cr}$ release assay using U-bottomed 96-well plates containing 5,000 targets/well. All assays were performed in duplicate. Percent cytotoxicity was determined from the formula: $100 \times[$ (experimental release
- spontaneous release)/(maximum release - spontaneous release $)]$. Maximum release was determined by lysis of targets by detergent ( $1 \%$ Triton X-100; Sigma Chemical Co.). Spontaneous release was $<20 \%$ of maximal release in all assays. The assay was considered positive if the specific ${ }^{51} \mathrm{Cr}$ release from target cells containing antigen was $\geq 15 \%$ higher than the nonspecific ${ }^{51} \mathrm{Cr}$ release from antigen negative target cells, and the nonspecific lysis was $<15 \%$ of maximum.

DNA-analysis. HBV-DNA was extracted from the serum as previously described, dissolved in TE buffer (15) and amplified in a programmable thermal cycler (Twin Block System; ERICOMP, Inc., San Diego, CA) by nested PCR. To amplify the region containing epitope Env250-258, Env260-269, Env335-343, Env348-357, Env370-379, Pol455-463, and Pol551-559 primer combination 1 or 2 was used. To amplify the region containing epitope Core 18-27 primer combination 3 or 4 was used (Table II). For each PCR the following program was used: $94^{\circ} \mathrm{C}$ for $4 \mathrm{~min}$ followed by 40 cycles of denaturation at $93^{\circ} \mathrm{C}$ for $5 \mathrm{~min}$, annealing at $56^{\circ} \mathrm{C}$ for $2 \mathrm{~min}$, and elongation at $72^{\circ} \mathrm{C}$ for $3 \mathrm{~min}$. The last cycle was followed by a 4-min extension step at $72^{\circ} \mathrm{C}$. The PCR products were analyzed by ethidium bromide-stained agarose gel electrophoresis. To eliminate sources of DNA contamination, DNA extraction and first PCR were performed in a separate room from the one used for handling of the amplified PCR products. Pipetting devices were kept separately and plugged pipette tips were used. All sera were divided into small aliquots under sterile conditions in a laminar flow hood and a separate aliquot was used for each analysis. All extractions and amplifications were performed in parallel with relevant negative and positive controls which consisted of DNA derived from the serum of healthy, uninfected individuals and HBV-positive patients. Contamination controls consisted of water added to the PCR mixture instead of DNA.

Direct sequencing of the PCR-products was performed with the PCR-product sequencing kit (Sequenase ${ }^{\mathrm{TM}}$; Amersham/USB, Arlington Heights, IL). Primer HBV-370S was used to sequence epitope Env250258, Env260-269, Pol455-463 in sense direction, primer HBV-536S to sequence epitope Env335-343, Env348-357, Env370-378, and Pol551559 in sense direction. Primer HBV-660AS was used to sequence epitope Env250-258, Env260-269, Pol455-463 in antisense direction and primer HBVSIAN to sequence epitope Env335-343, Env348-357, Env370-378, Pol551-559 in antisense direction. Epitope Core18-27 was 


\begin{tabular}{|c|c|c|}
\hline & Sequence & Position \\
\hline \multicolumn{3}{|c|}{ Primer combination 1 : } \\
\hline HBV-246S & 5'-GAGTCTAGACTCGTGGTGGA & $246-265$ \\
\hline HBV-1086AS & 5'-GCGAGAAAGTGAAAGCCTG & $1086-1105$ \\
\hline \multicolumn{3}{|l|}{ Internal primers } \\
\hline HBV-370S & 5'-TATCGCTGGATGTGTCTGC & $370-388$ \\
\hline HBV-HBVSIAN & 5'-TTTAGAGAGTAACCCCATCT & $851-870$ \\
\hline \multicolumn{3}{|c|}{ Primer combination 2: } \\
\hline HBV2830S & 5'-GAACAAGATCTACAGCATGG & $2830-2849$ \\
\hline HBV-1086AS & 5'-GCGAGAAAGTGAAAGCCTG & $1086-1105$ \\
\hline \multicolumn{3}{|l|}{ Internal primers } \\
\hline $\mathrm{HBV}-150 \mathrm{~S}$ & 5'-AACATGGAGAACATCACATC & $150-169$ \\
\hline HBV-HBVSIAN & 5'-TTTAGAGAGTAACCCCATCT & $851-870$ \\
\hline \multicolumn{3}{|l|}{ Sequencing primers: } \\
\hline \multicolumn{3}{|l|}{ Sense: } \\
\hline HBV-150S & 5'-AACATGGAGAACATCACATC & $150-169$ \\
\hline HBV-370S & 5'-TATCGCTGGATGTGTCTGC & $370-388$ \\
\hline HBV-536S & 5'-TCAAGGAACCTCTATGTATC & $536-555$ \\
\hline \multicolumn{3}{|l|}{ Antisense: } \\
\hline HBV-353AS & 5'-AGCGATAACCAGGACAAGTT & $353-372$ \\
\hline HBV-660AS & 5'-CTGAGCCAGGAGAAACGG & $660-678$ \\
\hline HBV-HBVSIAN & 5'-TTTAGAGAGTAACCCCATCT & $851-870$ \\
\hline \multicolumn{3}{|c|}{ Primer combination 3: } \\
\hline \multicolumn{3}{|c|}{ External primers } \\
\hline HBV-XISE & 5'-CAAGGTCTTACATAAGAGGA & $1641-1660$ \\
\hline HBV-2860AS & 5'-CAGAGGATTGCTGGTGGA & $2861-2878$ \\
\hline \multicolumn{3}{|l|}{ Internal primers } \\
\hline HBV-X2SE & 5'-CTCTTGGACTCTCAGCAATG & $1661-1680$ \\
\hline HBV-2627AS & 5'-CCTGGCAGGCATAATCAATT & $2627-2646$ \\
\hline \multicolumn{3}{|c|}{ Primer combination 4: } \\
\hline \multicolumn{3}{|c|}{ External primers } \\
\hline HBV-X2SE & 5'-CTCTTGGACTCTCAGCAATG & $1661-1680$ \\
\hline HBV-CBHINT & 5'-TTGCCTGAGTGCAGTATGGTGAGG & $2046-2069$ \\
\hline \multicolumn{3}{|l|}{ Internal primers } \\
\hline HBV-PCO & 5'-GGGAGGAGATTAGGTT & $1742-1757$ \\
\hline HBV-COR & 5'-AACATTGAGATTCCCGA & $2432-2448$ \\
\hline \multicolumn{3}{|l|}{ Sequencing primers: } \\
\hline \multicolumn{3}{|l|}{ Antisense: } \\
\hline HBV-CBHINT & 5'-TTGCCTGAGTGCAGTATGGTGAGG & $2046-2069$ \\
\hline HBV-NCO & 5'-CGGAAGTGTTGATA & $2317-2330$ \\
\hline HBV-COR & 5'-AACATTGAGATTCCCGA & $2432-2448$ \\
\hline
\end{tabular}

sequenced with primer HBV-CBHINT and the rest of the Core region was sequenced with primers HBV-COR and HBV-NCO in antisense direction. The predominant sequence was defined by the major band in the sequencing lanes when PCR-products were sequenced or by the sequence of $>50 \%$ of 5 to 12 independent clones. As shown by others (16) a viral subpopulation as low as $10 \%$ of the total population can be detected by direct sequencing of PCR-products. In selected cases, the PCR product was subcloned into the $\mathrm{PCR}^{\mathrm{TM}}$-vector with the TA Cloning Kit (Invitrogen, San Diego, CA). Cultures from individual colonies were grown overnight, plasmid was isolated with the QIAGEN Miniprep Kit (QIAGEN, Chatsworth CA) and 6-12 clones derived from at least two independent PCR reactions were sequenced.

Each patient's sequence was compared to the HBV sequences HPBAYWCI, HPBHBVAA, HPBVAYWE, HPBV, HPBVAYWC, HPBAYW, HPBADWZCG, HPBADW, HPBADW1, HPBHEPB,
HPBADW2, HPBADWZ, HPBADW3, HPBADRCG, HPBADRC, HPBADRA, HPBCGADR, HPBVADRM, HPBADR, and HPBAYR published in GenBank and to their consensus sequence. If patients were studied sequentially the HBV sequence was compared to the sequence derived from earlier serum samples from the same patient.

\section{Results}

Analysis of the CTL response to a panel of $T$ cell epitopes in acute and chronic $H B V$ infection. In this study we analyzed the CTL response to and the deduced amino acid sequence of 8 independent CTL epitopes in 6 patients with acute hepatitis and 12 patients with chronic hepatitis. The epitopes were chosen because they are located in three different HBV proteins, 


\begin{tabular}{|c|c|c|c|c|c|c|c|}
\hline \multirow[b]{2}{*}{ Epitope ${ }^{\circ}$} & \multirow[b]{2}{*}{ Peptide sequence* } & \multicolumn{3}{|c|}{ Conservation in } & \multirow{2}{*}{$\begin{array}{l}\text { Alternative peptide } \\
\text { sequences in } \\
\text { GenBank databases" }\end{array}$} & \multirow{2}{*}{$\begin{array}{l}\text { Alternative peptide } \\
\text { sequences as found } \\
\text { in patients' }\end{array}$} & \multirow[b]{2}{*}{ Patients** } \\
\hline & & GenBank $^{\ddagger}$ & Acutes ${ }^{8}$ & Chronics $^{8}$ & & & \\
\hline Core18-27 & FLPSDFFPSV & $45 \%$ & $100 \%$ & $92 \%$ & $\begin{array}{l}\text { FLPSDFFPSI } \\
\text { YLPSDFFLYYV }\end{array}$ & FLPSDFFPSI & C-9 \\
\hline Env250-258 & LLLCLIFLL & $100 \%$ & $100 \%$ & $100 \%$ & none & none & \\
\hline Env260-269 & LLDYQGMLPV & $90 \%$ & $83 \%$ & $100 \%$ & LLDYQGMLHV & none & \\
\hline Env335-343 & WLSLLVPFV & $100 \%$ & $100 \%$ & $100 \%$ & none & none & \\
\hline Env348-357 & GLSPTVWLSV & $75 \%$ & $33 \%$ & $42 \%$ & $\begin{array}{l}\text { GLSPTVWLSA } \\
\text { GLSPTVWSSSV }\end{array}$ & $\begin{array}{l}\text { GLSPTVWLSA } \\
\text { GLSPTVWPSA }\end{array}$ & $\begin{array}{l}\text { A-2,A-4,A-5,C-3,C-5,C-6,C-7,C-8,C-11 } \\
\text { C-4 }\end{array}$ \\
\hline Env370-379 & SIVSPFIPLL & $10 \%$ & $50 \%$ & $33 \%$ & $\begin{array}{l}\text { SILSPFLPLL } \\
\text { SIVSSFIPLL } \\
\text { TILSPFLPLL } \\
\text { NIILSPFMPLL }\end{array}$ & $\begin{array}{l}\text { SILSPFLPLL } \\
\text { NILSPFLPLL } \\
\bar{N} \overline{\text { ILSPFIPLL }} \\
\overline{\text { NIVSSPFIPLL }} \\
\overline{\text { SIVSPFLPLL }}\end{array}$ & $\begin{array}{l}\text { C-2 } \\
\text { C-6 } \\
\text { C-4,C-5,C-8,C-9,C-10 } \\
\text { A-2 } \\
\text { A-1,A-4,C-7 }\end{array}$ \\
\hline Pol455-463 & GLSRYVARL & $55 \%$ & $100 \%$ & $92 \%$ & GLPRYVARL & GLPRYVARL & $\mathrm{C}-3$ \\
\hline Pol551-559 & YMDDVVLGA & $90 \%$ & $100 \%$ & $100 \%$ & YMDDMVLGA & none & \\
\hline
\end{tabular}

* Amino Acid Sequence of epitope peptides used to stimulate CTL responses in PBMC. ${ }^{\ddagger}$ Frequency (\%) at which the epitope peptide sequence is present in the HBV isolated published in GenBank. ${ }^{\S}$ Frequency (\%) at which the epitope peptide sequence is present in the patients studied.

"Alternative peptide sequences at the corresponding locations in the GenBank database. "Actual viral peptide sequences detected in patients included in this study. ** Patients in whom the variant sequences were identified.

namely core, envelope, and polymerase, and because they have been shown to expand in vivo-primed polyclonal CTL from patients with acute hepatitis $B(1,5,6)$. As we have previously reported, this CTL response is multispecific, i.e., targeted against multiple epitopes in all viral proteins, and it can persist for 6-18 mo after recovery from acute hepatitis B infection, and cytotoxic $\mathrm{T}$ lymphocytes against all of these epitopes have been shown to be CD8 positive and HLA-A2 restricted $(1,5$, 6, Chisari, F. V., unpublished observations). The degree of sequence conservation within these epitopes varies from 10$100 \%$ of the sequences present in the GenBank Data Base (Table III).

As shown in Fig. 1, all of the 6 acutely infected patients responded to two or more of the epitopes tested. A total of 23 CTL responses were detected out of a possible $43(53 \%)$ in this group of patients. CTL specific for these epitopes have previously been shown to recognize target cells pulsed with very low concentrations $(0.001 \mu \mathrm{M})$ of the inducing peptide, but they do not recognize irrelevant peptides even at high concentrations $(10 \mu \mathrm{M})(1,5,6)$. Their relevance during an acute infection is supported by the fact, that such CTL have also been shown to lyse target cells presenting endogenously processed antigen $(1,5,6)$.

In contrast, 8 of the 12 chronically infected patients failed to respond to any of the epitopes, and the remaining 4 patients produced only weak responses to a single epitope, such that only 5 out of $96(5 \%)$ possible CTL responses were observed in these chronically infected patients. To examine the possibility that chronically infected patients responded to a different set of peptides from the acutely infected patients, we stimulated PBMC from a subset of these patients with an extended panel of HBV-derived peptides with high to intermediate HLA-A2 binding affinity in the range which we have previously shown to be required for immunogenicity (17). We have previously shown this panel of peptides to be nonstimulatory to acutely infected patients (17). As shown in Table IV, no CTL responses were generated to any of these peptides by these patients.
Analysis of the T cell epitope sequence of the infecting virus in acute and chronic HBV infection. As stated above, a narrowly focused CTL response, that might exert selective pressure on the virus, was observed in 5 out of $96 \mathrm{CTL}$ assays in chronically infected patients (Fig. 1). In each instance, however, only prototype epitope sequences matching the ones in the peptides were observed (Table V). Similarly, prototype epitope sequences were present in 23 out of 23 instances $(100 \%)$ where a strong CTL response was observed in the acute HBV patients. This suggests that CTL pressure was not sufficient to select for escape mutants at these loci in these patients. Additionally, since an equal fraction of the examined epitopes $(\sim 20 \%)$ were different from the stimulating peptide in both groups of patients, and since this was always associated with nonresponsiveness at these loci, it is possible that the patients had not been primed in vivo to these epitopes. This should not be a cause of persistent infection, however, since it was seen in only a few isolated and randomly distributed epitopes, and it was as common in patients who cleared the virus as it was in those who did not ( Table III).

CTL response to alternate viral epitope sequences. To understand whether the patients infected by viral variants who did not respond to the prototype peptide could respond to the variant peptides, we stimulated the PBMC from patient C-2 and C-6 who failed to respond to Env 370-378 with the peptides corresponding to the viral sequence with which they were infected (Table III). In both peptides the HLA-A2-binding motif was still present. No specific cytotoxicity was observed against the prototype or the variant peptides in either patient (not shown) suggesting that the variant sequences were not selected by immune pressure and that they were either present in the virus at the time of infection or they were selected for other reasons. Alternatively, the CTL response against the prototype sequence could have vanished and the new variant epitope could be unable to stimulate a new CTL response.

Sequential analysis of epitope sequence and CTL response during chronic HBV infection. Patients C-4, C-6, and C-3 with 


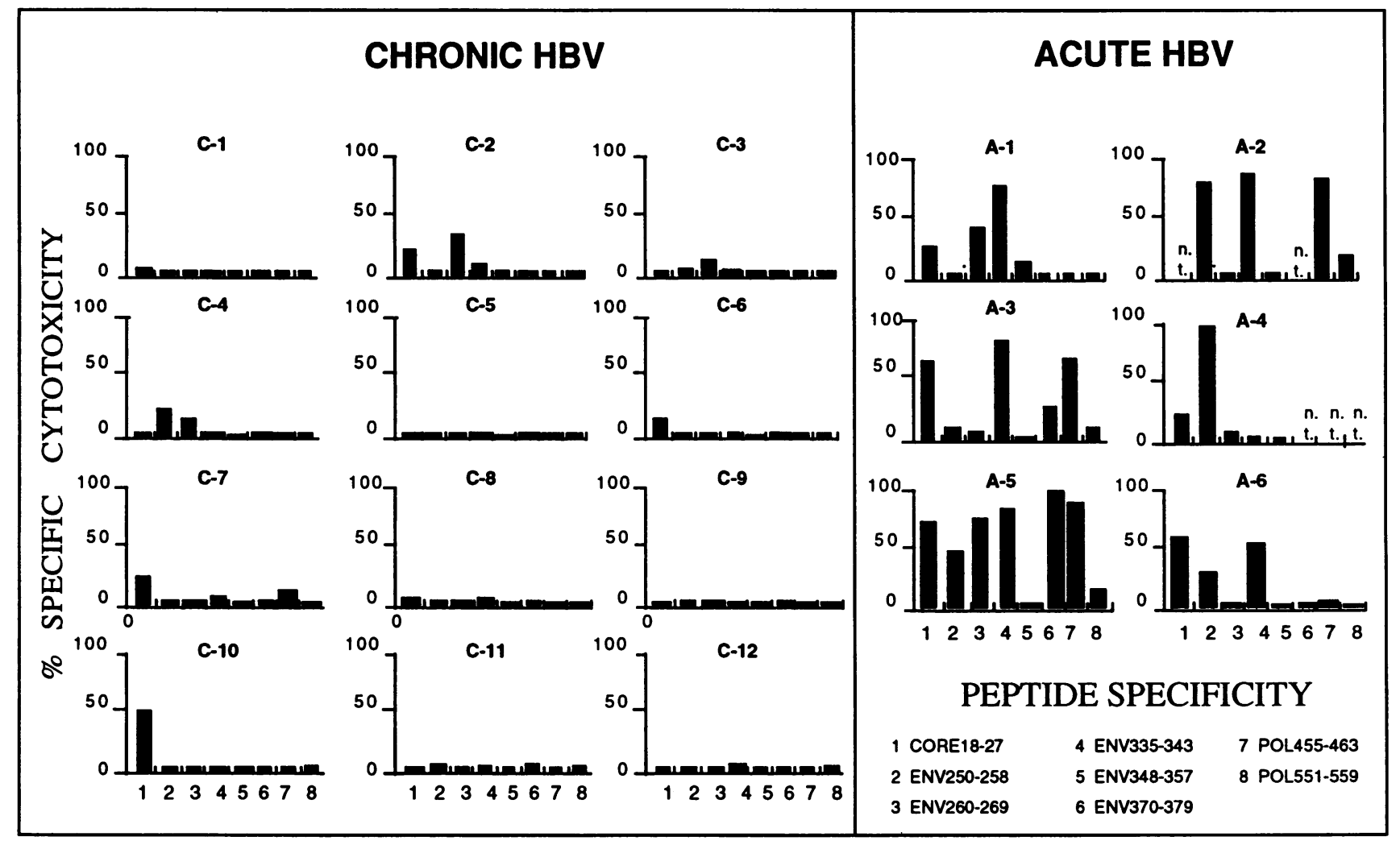

Figure 1. The CTL response in acute and chronic HBV infection: HBV-specific CTL response in patients with chronic hepatitis $(C-1-C-9)$ and acute hepatitis (A-1-A-9). PBMC were stimulated with $10 \mu \mathrm{g}$ of the peptides indicated for $2 \mathrm{wk}$ as described in Methods, and tested in a 4-h ${ }^{51} \mathrm{Cr}$ release assay against JY target cells prepulsed overnight with the same peptide. Peptide-specific cytotoxicity was measured by subtracting the ${ }^{51} \mathrm{Cr}$ release by JY target cells not prepulsed with the peptide from the ${ }^{51} \mathrm{Cr}$ release by JY target cells prepulsed with $10 \mu \mathrm{M}$ of the peptide. Results shown represent percent specific lysis in a 4-h ${ }^{51} \mathrm{Cr}$ release by JY target cells prepulsed with $10 \mu \mathrm{M}$ of the peptide at an Effector: Target Ratio of 50:1 (n.t., not tested).

chronic HBV infection were followed over 10, 29, and 55 mo, respectively. In patient $\mathrm{C}-3$ a sequence change was found in the HLA-A2.1-binding residue of the core18-27 epitope of serum-derived HBV-DNA, so this patient was analyzed sequentially (Table VI). Synthetic peptides corresponding to the original and the alternate sequence were used to stimulate peripheral blood lymphocytes and to test for specific cytotoxicity against JY-EBV cells pulsed with the corresponding peptide. No immune response to either the original or alternate sequence was observed.

Analysis of sequence variability in the core mutation "cluster" region in chronically infected patients. To compare the degree of sequence heterogeneity in our patients with that reported previously in patients with active liver disease from Japan (13) and China (14), we sequenced the first 139 codons of the core region which contain two of the three mutation "cluster" regions defined by those investigators (i.e., codons 48-60 and 84-101). We compared the HBV nucleotide sequence derived from each patient with the HBV sequences HPBAYWCI, HPBHBVAA, HPBVAYWE, HPBV, HPBVAYWC, HPBAYW, HPBADWZCG, HPBADW, HPBADW1, HPBHEPB, HPBADW2, HPBADWZ, HPBADW3, HPBADRCG, HPBADRC, HPBADRA, HPBCGADR, HPBVADRM, HPBADR, and HPBAYR published in GenBank. The comparison with isolate HPBADR and the previously published data is shown in Fig. 2. In contrast to the previous reports $(13,14)$ in Eastern patients we do not find clustered mutations in codon
48-60 and 84-101. The number of divergent amino acids is even less in our patient group if subtype HPBADW, which is more frequent in Western patients, is chosen for amino acid comparison. In this case there will be no amino acid differences at position $75,77,87$, and 91 of the core protein (marked by an asterisk in Fig. 2).

Comparison of sequence variability in intraepitope and interepitope regions in acute and chronic $\mathrm{HBV}$ infection. When each patient's HBV sequence was compared to the HBV sequences published in GenBank no complete match was found. Each patient-derived HBV sequence shared between 89 and $100 \%$ of the nucleotides with each of the GenBank HBV sequences. To standardize the analysis of intra- and interepitope variability, the patient-derived HBV sequences were compared to the consensus sequence of the GenBank HBV isolates. As shown in Table VII, the sequence variability in both intra- and interepitope regions in the chronically infected patients was the same as in the acutely infected patients. Importantly, at these epitope sites in both patient populations the intraepitope regions display no greater sequence variability than the interepitope region of the virus suggesting that CTL selection pressure at these epitope sites is absent in these patients.

\section{Discussion}

In this study, we demonstrated that CTL escape mutations are not commonly observed in viral sequences in patients with 


\begin{tabular}{|c|c|c|c|c|c|c|}
\hline \multirow[b]{2}{*}{ Protein } & \multirow[b]{2}{*}{ Amino acid location } & \multirow[b]{2}{*}{ Epitope sequence } & \multirow[b]{2}{*}{ HLA-A2.1-binding affinity } & \multirow[b]{2}{*}{ Percent conserved* } & \multicolumn{2}{|c|}{ No. Positive/No. Tested } \\
\hline & & & & & CTL-assays ${ }^{\ddagger}$ & Patients ${ }^{8}$ \\
\hline Env & $371-379$ & ILSPFLPLL & 2.9000 & 65 & $0 / 24$ & $0 / 3$ \\
\hline Core & $118-126$ & LVSFGVWI & 2.7000 & 90 & $0 / 24$ & $0 / 3$ \\
\hline Env & $338-347$ & LLVPFVQWFV & 1.6000 & 95 & $0 / 24$ & $0 / 3$ \\
\hline Pol & $527-535$ & LLAQFTSAI & 0.7100 & 100 & $0 / 48$ & $0 / 3$ \\
\hline Env & $259-268$ & VLLDYQGMLPSV & 0.7000 & 90 & $0 / 24$ & $0 / 3$ \\
\hline Env & $360-368$ & MMWYWGPSL & 0.4100 & 85 & $1 / 24$ & $1 / 3$ \\
\hline Env & $249-258$ & ILLLCLIFLL & 0.3000 & 100 & $0 / 24$ & $0 / 3$ \\
\hline Env & $348-357$ & GLSPTVWLSV & 0.2800 & 75 & $0 / 52$ & $0 / 4$ \\
\hline Core & $18-27$ & FLPSDFFPSI & 0.2100 & 50 & $0 / 24$ & $0 / 3$ \\
\hline Pol & $655-663$ & ALMPLYACI & 0.2000 & 100 & $0 / 24$ & $0 / 3$ \\
\hline Core & $59-71$ & ILCWGELMTL & 0.1900 & 45 & $0 / 24$ & $0 / 3$ \\
\hline Env & $188-195$ & VLQAGFFLL & 0.1500 & 95 & $0 / 24$ & $0 / 2$ \\
\hline Pol & $63-71$ & GLYSSTVPV & 0.1500 & 60 & $0 / 24$ & $0 / 2$ \\
\hline Pol & $504-512$ & HLYSHPIIL & 0.1300 & 84 & $0 / 40$ & $0 / 4$ \\
\hline Env & $259-267$ & VLLDYQGML & 0.1100 & 95 & $0 / 24$ & $0 / 2$ \\
\hline Env & $378-388$ & LLPIFFCLWV & 0.1000 & 100 & $0 / 24$ & $0 / 2$ \\
\hline Env & $260-270$ & LLDYQGMLPV & 0.0850 & 90 & $0 / 24$ & $0 / 2$ \\
\hline Env & $359-268$ & WMMWYWGPSL & 0.0840 & 85 & $0 / 24$ & $0 / 2$ \\
\hline Pol & $816-824$ & SLYAVSPSV & 0.0780 & 56 & $0 / 40$ & $0 / 4$ \\
\hline Env & $251-259$ & LLCLIFLLV & 0.0490 & 100 & $0 / 40$ & $0 / 4$ \\
\hline PreCore & $2-10$ & QLFHLCLII & 0.0390 & 88 & $0 / 24$ & $0 / 2$ \\
\hline Env & $249-257$ & ILLLCLIFL & 0.0350 & 100 & $0 / 24$ & $0 / 2$ \\
\hline Env & $175-184$ & LLVLQAGFFL & 0.0310 & .90 & $0 / 24$ & $0 / 2$ \\
\hline Env & $377-385$ & PLLPIFFCL & 0.0310 & 100 & $0 / 24$ & $0 / 2$ \\
\hline Env & $248-257$ & FILLLCLIFL & 0.0280 & 80 & $0 / 40$ & $0 / 4$ \\
\hline Core & $100-108$ & LLWFHISCL & 0.0240 & 90 & $0 / 40$ & $0 / 4$ \\
\hline Core & $139-148$ & ILSTLPETTV & 0.0220 & 100 & $0 / 40$ & $0 / 4$ \\
\hline Pol & $773-782$ & ILRGTSFVYV & 0.0160 & 89 & $0 / 40$ & $0 / 4$ \\
\hline Env & $176-184$ & LVLQAGFFL & 0.0150 & 90 & $0 / 40$ & $0 / 4$ \\
\hline Pol & $765-773$ & LLGCAANWI & 0.0140 & 89 & $0 / 40$ & $0 / 4$ \\
\hline Core & $99-108$ & QLLWFHISCL & 0.0130 & 90 & $0 / 40$ & $0 / 4$ \\
\hline Pol & $424-432$ & NLSWLSLDV & 0.0130 & 90 & $0 / 40$ & $0 / 4$ \\
\hline
\end{tabular}

* Percentage of HBV sequences containing the precise epitope in GenBank database; ${ }^{\ddagger} \mathrm{CTL}$ assay rated positive, if specific cytotoxicity $>15 \%$ lysis; ${ }^{8}$ patient rated positive if one CTL assay was rated positive.

chronic HBV infection. To examine this question, we compared the DNA sequence of, and the CTL response to, eight HLAA2-restricted CTL epitopes in the HBV nucleocapsid, envelope, and polymerase proteins in 18 HLA-A2-positive patients with acute $(n=6)$ and chronic hepatitis $\mathrm{B}(n=12)$. We report that the absence of a detectable CTL response in the majority of patients with chronic hepatitis B virus infection in the current

Table V. Analysis of CTL Epitope Sequence and CTL Response

\begin{tabular}{lccccc}
\hline & & \multicolumn{4}{c}{ Peptide sequence } \\
\cline { 3 - 6 } Category & CTL & response & \multicolumn{2}{c}{ Indentical } & \multicolumn{2}{c}{ Nonidentical } \\
\hline \multirow{4}{*}{ Acute } & & Responders & Nonresponders & Responders & Nonresponders \\
& $23 / 43$ & $23 / 23$ & $16 / 20$ & $0 / 23$ & $4 / 20$ \\
Chronic & $(53 \%)$ & $(100 \%)$ & $(80 \%)$ & $(0 \%)$ & $(20 \%)$ \\
& $5 / 96$ & $5 / 5$ & $74 / 91$ & $0 / 5$ & $17 / 91$ \\
& $(5 \%)$ & $(100 \%)$ & $(81 \%)$ & $(0 \%)$ & $(19 \%)$ \\
\hline
\end{tabular}

study is not due to infection by viruses that contain variant CTL epitopes. In the few instances where a narrowly focused CTL response was present in the chronically infected patients, it did not lead to the emergence of CTL escape mutants. Nucleotide and amino acid sequences were not more variable within the CTL epitopes than within viral regions external to the epitopes, and the entire $\mathrm{HBV}$ core protein displayed the same degree of amino acid heterogeneity as the rest of the sequenced virus in these patients. Furthermore, the amino acid sequence heterogeneity of the virus present in the persistently infected patients was no greater than that seen in acutely infected patients who successfully cleared the virus. Finally, in a chronically infected patient who developed an amino acid change in the HLA-A2binding residue of one of these epitopes, no immune response was detectable to either the original sequence or to the emerged variant indicating that variation can occur within well-defined CTL epitopes in the absence of immune selection pressure.

We conclude that the weak HBV-specific CTL response we observed in these chronically infected patients is the primary determinant of viral persistence, and that in general the CTL response is too weak in most chronically infected patients to 
Table VI. Epitope Sequence Change and Immune Response

\begin{tabular}{lccc}
\hline & Sequencing analysis & \multicolumn{2}{c}{ Immunological analysis } \\
\hline & HBV-epitope & \multicolumn{2}{c}{ Specific cytotoxicity } \\
Date & sequence in serum & FLPSDFFPSI* & FLPSDFFPSV* \\
& & & \\
$11 / 06 / 87$ & FLPSDFFPSI & 0 & n.t. \\
$02 / 05 / 88$ & I & 0 & n.t. \\
$09 / 22 / 89$ & I & 1 & n.t. \\
$12 / 15 / 89$ & I & 2 & n.t. \\
$08 / 01 / 90$ & I & n.t. & 0 \\
$08 / 30 / 91$ & V & 0 & 0 \\
$04 / 17 / 92$ & V & 0 & 2 \\
$06 / 08 / 92$ & V & 3 & 3 \\
& & & \\
\hline
\end{tabular}

* Peptide used to expand CTL and to pulse JY-EBV used as targets in the CTL assay. n.t., not tested.

select for the emergence of escape mutants at the epitope sites studied. Since we limited our analysis to eight epitopes previously defined in patients with acute hepatitis we cannot completely exclude the possibility that CTL responses to different epitopes might occur in patients with chronic infection and that these responses might select CTL escape mutants. We believe, however, that CTL escape mutations are unlikely to be a fre-

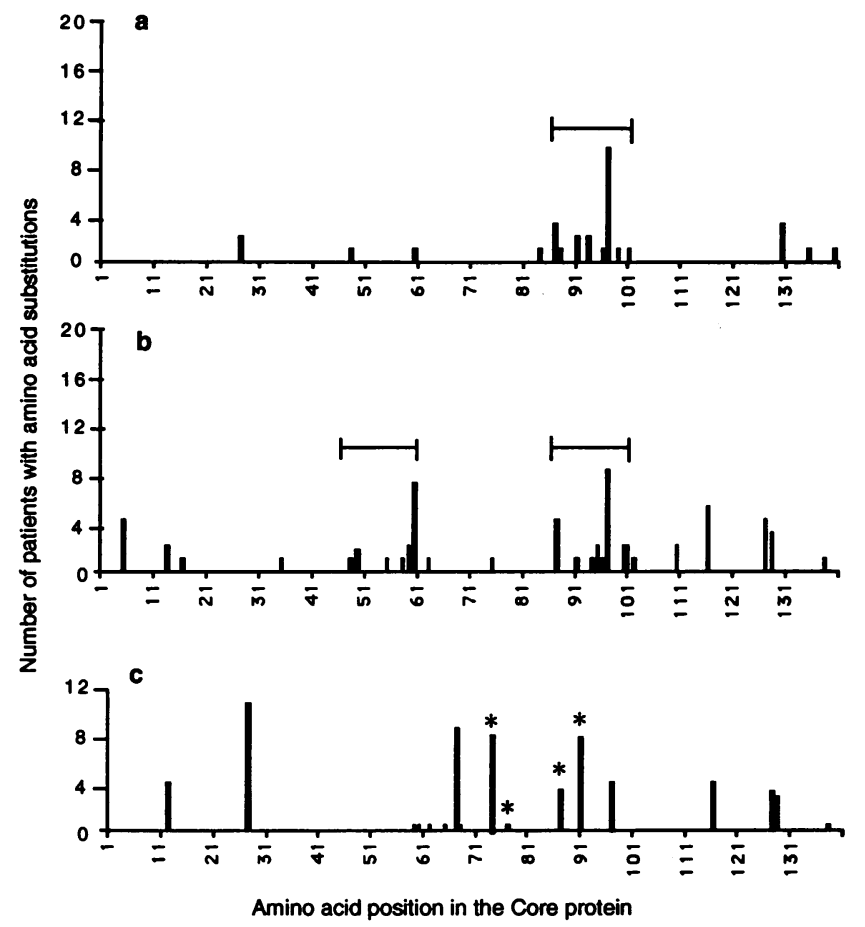

Figure 2. Amino acid variability of the Core protein. The number of patients with amino acid substitutions at each position of the Core protein is given for the study of Ehata et al. (a) (13), Chuang et al. (b) (14), and this study (c). The amino acid sequence is compared to the HPBADR subtype to allow comparison with the two previously published studies $(13,14)$. As the current study $(c)$ analyzes a Western patient population, which is most likely infected by the HPBADW subtype, amino acids that do not match with HPBADR, but do match with HPBADW are marked with an asterisk. The mutation cluster regions (amino acid 48-60 and 84-101) are denoted by a horizontal bar above the corresponding positions.
Table VII. Sequence Variability in Different Regions of the Hepatitis B Virus

\begin{tabular}{lcc}
\hline & $\begin{array}{c}\text { Amino acids } \\
\text { analyzed* }\end{array}$ & $\begin{array}{c}\text { Percent homology with GenBank } \\
\text { HBV consensus sequence }\end{array}$ \\
\hline Chronic HBV patients & & \\
$\quad$ Region with epitopes & 912 & 97.8 \\
$\quad$ Region without epitopes & 4580 & 94.4 \\
Acute HBV patients & & \\
Region with epitopes & 456 & 98.7 \\
Region without epitopes & 794 & 95.6 \\
\hline
\end{tabular}

* The number of amino acids analyzed refers to all of the 12 patients with chronic hepatitis B and all of the 6 patients with acute hepatitis $B$, respectively. The number of amino acids in regions without epitopes also considers translations according to different open reading frames.

quent event for several reasons: first, all the epitopes we studied are commonly recognized by patients with acute hepatitis B who successfully clear the virus. Second, we failed to observe CTL responses to an extended panel of 32 additional HBVderived peptides that bind well to HLA-A2. Moreover, the existence of a completely different set of subdominant epitopes in patients with chronic HBV infection has not been demonstrated at this point in time. Third, in the two previously published cases $(10,11)$ in which viral escape may have indeed occurred, the narrowly focussed immune response targeted against one of these epitopes was uncommonly strong for chronically infected patients in our experience. Fourth, the rarity of CTL escape at the epitope described in these patients is underscored by the absence of variation in that sequence in the $20 \mathrm{HBV}$ isolates catalogued in the GenBank database. Nonetheless, until it becomes technically possible to study the CTL response to full length viral proteins in HBV-infected individuals, the possibility that CTL responses to currently unidentified epitopes can effectively select for escape variants remains possible. Based on the currently available data, however, we propose that selection of CTL escape mutants is probably an unusual event during chronic HBV infection and that it probably occurs only when the CTL response is stronger than usual but narrowly focussed $(8,9)$.

The current data suggest that if CTL "escape" mutations occur in $\mathrm{HBV}$, they could contribute to its persistence, but they probably do not represent its primary cause in the majority of patients where the precipitating factor would appear to be a weak immune response. Indeed, the absence of viral escape mutants in the current study is additional evidence that the CTL response in most chronically infected patients is too weak to select for variants.

Several other mechanisms might explain viral persistence in chronically infected patients. These include neonatal tolerance, immunological exhaustion by large viral loads, infection of immunologically privileged sites, modulation of recognition molecules on the surface of infected cells, etc. We favor the idea that the outcome may in fact be determined very soon after infection by the relative kinetics of viral spreading and induction of the immune response. According to this scenario a rapid and vigorous immune response would lead to viral clearance. In contrast, if the immune response is relatively slow to begin, the number of virus-infected hepatocytes may become so high that immune-mediated viral clearance could be impossible even if 
the late-developing CTL response is vigorous. In this setting, viral mutations that abrogate or antagonize $\mathrm{T}$ cell recognition could be selected, but even under these circumstances the immune response to all of the remaining viral epitopes would have to be unable to clear the virus for selection to occur.

Finally, the current data demonstrate that comparisons between individual single viral isolates and consensus sequences are not likely to reflect mutations that have occurred in that patient unless they are located within otherwise conserved regions of the virus. Furthermore, these results illustrate that the only definitive evidence of viral escape mutation is demonstration of an amino acid sequence change in a specific epitope in two sequential samples from an individual patient combined with loss of recognition of that epitope by CTL derived from the same patient. Unless this kind of combined analysis of viral sequence and $\mathrm{T}$ cell responsiveness is performed at multiple time points in individual patients, one should not conclude that variant viral sequences have been selected by immune pressure.

\section{Acknowledgments}

We would like to thank our clinical collaborators who have provided patient material for this study over the past several years, especially Dr. Ernest Ribera and John Person (Naval Research Medical Center, San Diego, CA), and Dr. Allan Redeker (Rancho Los Amigos Medical Center, USC Liver Unit, Downey, CA).

This work was supported by U. S. Public Health Service grant AI20001 and grant RR00833 from the National Institutes of Health. B. Rehermann was supported by grant Re 1017/1-1 of the Deutsche Forschungsgemeinschaft, Bonn, Germany. This is manuscript number 9173-MEM from the Scripps Research Institute.

\section{References}

1. Bertoletti, A., C. Ferrari, F. Fiaccadori, A. Penna, R. Margolskee, H. J. Schlicht, P. Fowler, S. Guilhot, and F. V. Chisari. 1991. HLA class I-restricted human cytotoxic $\mathrm{T}$ cells recognize endogenously synthesized hepatitis B virus nucleocapsid antigen. Proc. Natl. Acad. Sci. USA. 88:10445-10449.

2. Penna, A., F. V. Chisari, A. Bertoletti, G. Missale, P. Fowler, T. Giuberti, F. Fiaccadori, and C. Ferrari. 1991. Cytotoxic T lymphocytes recognize an HLAA2 restricted epitope within the hepatitis B virus nucleocapsid antigen. J. Exp. Med. 174:1565-1570.

3. Bertoletti, A., F. V. Chisari, A. Penna, S. Guilhot, L. Galati, P. Fowler, A. Vitiello, R. C. Chesnut, F. Fiaccadori, and C. Ferrari. 1993. Definition of a minimal optimal cytotoxic $\mathrm{T}$ cell epitope within the hepatitis B virus nucleocapsid protein. J. Virol. 67:2376-2380.

4. Missale, G., A. Redeker, J. Person, P. Fowler, S. Guilhot, H. J. Schlicht C. Ferrari, and F. V. Chisari. 1993. HLA-A31 and Aw68 restricted cytotoxic T cell responses to a single hepatitis $\mathrm{B}$ virus nucleocapsid epitope during acute viral hepatitis. J. Exp. Med. 177:751-762.

5. Nayersina, R., P. Fowler, S. Guilhot, G. Missale, A. Cerny, H. J. Schlicht, A. Vitiello, R. Chesnut, J. L. Person, A. G. Redeker and F. V. Chisari. 1993. HLA-A2 restricted cytotoxic T lymphocyte response to multiple hepatitis B surface antigen epitopes during hepatitis B virus infection. J. Immunol. 150:46594671 .

6. Rehermann, B., P. Fowler, J. Sidney, J. Person, A. Redeker, M. Brown, B. Moss, A. Sette, and F. V. Chisari. 1995. The cytotoxic T lymphocyte response to multiple hepatitis B virus polymerase epitopes during and after acute viral hepatitis. J. Exp. Med. 181:1047-1058.

7. Barnaba, V., A. Franco, A. Alberti, C. Balsano, R. Benvenuto, F. Balsano. 1989. Recognition of hepatitis B virus envelope proteins by liver-infiltrating $T$ lymphocytes in chronic HBV infection. J. Immunol. 143:2650-2655.

8. Ferrari, C., A. Penna, T. Giuberti, M. J. Tong, E. Ribera, F. Fiaccadori, and F. V. Chisari. 1987. Intrahepatic, nucleocapsid antigen-specific $T$ cells in chronic active hepatitis B. J. Immunol. 139:2050-2058.

9. Pircher, H., D. Moskophidis, U. Rohrer, K. Bürki, H. Hengartner, and R. M. Zinkernagel. 1990. Viral escape by selection of cytotoxic $\mathrm{T}$ cell-resistant virus variants in vivo. Nature (Lond.). 346:629-633.

10. Bertoletti, A., A. Costanzo, F. V. Chisari, M. Levrero, M. Artini, A. Sette, A. Penna, T. Giuberti, F. Fiaccadori and C. Ferrari. 1994. Cytotoxic T lymphocyte response to a wild type hepatitis B virus epitope in patients chronically infected by variant viruses carrying substitutions within the epitope. J. Exp. Med. 180:933943.

11. Bertoletti, A., A. Sette, F. V. Chisari, A. Penna, M. Levrero, M. De Carli, F. Fiaccadori, and C. Ferrari. 1994. Natural variants of cytotoxic epitopes are Tcell receptor antagonists for antiviral cytotoxic T cells. Nature (Lond.). 369:407410.

12. Wakita, T., S. Kakumu, M. Shibata, K. Yoshioka, Y. Ito, T. Shinagawa, T. Ishikawa, M. Takayanagi, and T. Morishima. 1991. Detection of pre-C and core region mutants of hepatitis B virus in chronic hepatitis B virus carriers. $J$. Clin. Invest. 88:1793-1801.

13. Ehata, T., M. Omata, W. L. Chuang, O. Yokosuka, Y. Ito, K. Hosoda and M. Ohto. 1993. Mutations in core nucleotide sequence of hepatitis B virus correlate with fulminant and severe hepatitis. J. Clin. Invest. 91:1206-1213.

14. Chuang, W.-L., M. Omata, T. Ehata, O. Yokosuka, Y. Ito, F. Imazeki, S.-N. Lu, W.-Y. Chang, and M. Ohto. 1993. Precore mutations and core clustering mutations in chronic hepatitis B virus infection. Gastroenterology. 104:263-271.

15. Michalak, T. I., C. Pasquinelli, S. Guilhot, and F. V. Chisari. 1994. Hepatitis B virus persists after recovery from acute viral hepatitis. J. Clin. Invest. 93:230-239.

16. Raimondo, G., M. Stemler, R. Schneider, G. Wildner, G. Squadrito, and H. Will. 1990. Latency and reactivation of a pre-core mutant hepatitis B virus in a chronically infected patient. J. Hepatol. 11:374-380.

17. Sette, A., A. Vitiello, B. Rehermann, P. Fowler, R. Nayersina, W. M. Kast, C. J. M. Melief, C. Oseroff, L. Yuan, J. Ruppert, et al. 1994. The relationship between class I binding affinity and immunogenicity of potential cytotoxic $\mathrm{T}$ cell epitopes. J. Immunol. 153:5587-5592. 